\title{
The usefulness of pedicled perforator flap in partial breast reconstruction after breast conserving surgery in Korean women
}

\author{
Jae Bong Kim ${ }^{1}$, Dong Kyu Kim ${ }^{1}$, Jeong Woo Lee ${ }^{1}$, Kang Young Choi ${ }^{1}$, Ho Yun Chung ${ }^{1}$, \\ Byung Chae Cho ${ }^{1}$, Ho Yong Park ${ }^{2}$, Jee Yeon Lee ${ }^{2}$, Jung Dug Yang ${ }^{1}$ \\ Departments of ${ }^{1}$ Plastic and Reconstructive Surgery and ${ }^{2}$ Surgery, Kyungpook National University School of Medicine, Daegu, Korea.
}

Background The emergence of breast-conserving surgery combined with radiotherapy as the treatment of choice for early stage breast cancer has resulted in greater focus on oncoplastic breast surgery. The use of perforator flaps has particularly gained in reputation for its effectiveness in the reconstruction of partial breast defects in Korean women. Herein, we present our experience with the use of thoracodorsal artery perforator (TDAP) and lateral intercostal artery perforator (LICAP) flaps.

Methods This study included 33 patients who underwent breast reconstruction using TDAP or LICAP flaps at our hospital from January 2011 to December 2014. Data from patient medical records, and patient satisfaction surveys, which were conducted 12 months postoperatively, were retrospectively evaluated.

Results TDAP and LICAP flap-based reconstructions were performed in 14 and 19 patients, respectively. Five patients developed complications that required additional intervention. Overall patient satisfaction was observed to be excellent in $15(46 \%)$ patients, and good in 12 $(36 \%)$.

Conclusions Based on our experience, oncoplastic breast surgery using TDAP or LICAP flap is an effective remodeling technique for small-to-moderate breast defects in Korean women with smaller breasts.

Keywords Intercostal artery perforator flap / Oncoplastic breast surgery / Thoracodorsal artery perforator flap / Latissimus dorsi flap / Breast reconstruction
Correspondence: Jung Dug Yang Department of Plastic and Reconstructive Surgery, Kyungpook National University School of Medicine, 130 Dongdeok-ro, Daegu 41944, Korea

Tel: +82-53-420-5681

Fax: +82-53-425-3879

E-mail: lambyang@knu.ac.kr

Received: 24 Jul $2017 \bullet$ Revised: 5 Oct $2017 \bullet$ Accepted: 10 Oct 2017

pISSN: 2234-6163・ elSSN: 2234-6171・https://doi.org/10.5999/aps.2017.01200 • Arch Plast Surg 2018;45:29-36

\section{INTRODUCTION}

Breast conserving surgery (BCS) with radiotherapy (RT) is reported to be similar to total mastectomy (TM) in terms of oncological safety, with the result that BCS with RT is being increasingly considered as the first choice of treatment for early stage breast cancer $[1,2]$. Such a shift may be influenced by the trend in oncology favoring minimally invasive excisions, as well as patients preferring better postoperative quality of life (QOL) [3]. Surgeons have attempted to develop various partial breast reconstruction techniques to improve the breast asymmetry (or overall body image) resulting from BCS, as measures to improve 
patients' QOL [4-6]. However, there is no consensus as to the best oncoplastic breast surgical technique for any given situation. Hence, surgeons make the decisions on reconstruction techniques based on breast size and the defect-to-breast-volume ratio following tumor excision.

Our hospital has made consistent efforts to establish algorithms for breast reconstruction techniques applicable to different types of patients, depending on the excised mass $[7,8]$. Since Korean women have smaller breasts than their western counterparts, lumpectomy often results in significant breast deformity as the residual breast tissue is minimal [7]. Volume replacement (VR) techniques can overcome this limitation and provide sufficient volume, and stable aesthetic results. However, these techniques may result in donor morbidity and require surgical expertise, thus limiting their application. Among the different VR techniques, the latissimus dorsi (LD) flap has been widely used for breast reconstruction following BCS in patients with smallto moderate-sized breasts. This flap can provide coverage for a large range of defects, both in terms of location and size. Nonetheless, the large size of the flap may contribute to postoperative breast asymmetry as well as donor site morbidity. Therefore, there is a need for newer flaps suitable for smaller defects. Hamdi et al. $[5,9,10]$ successfully proved the effectiveness of perforator flaps, in particular the thoracodorsal artery perforator (TDAP) flap and the intercostal artery perforator (ICAP) flap, and demonstrated their aesthetic and functional excellence $[11,12]$.

Although breast reconstruction is performed to improve patients' QOL, the outcomes are not always satisfactory as described in the literature, and according to our own experience as well; there were cases where patient satisfaction with cosmetic outcomes was as low as 2 or 3 on a 10 -point scale $[13,14]$. The choice of reconstruction technique is therefore crucial, along with the need for extensive information sharing between surgeons. We present our experience of 33 cases of breast recon- struction performed using the TDAP or lateral ICAP (LICAP) flap, in Kyungpook National University Hospital from January 2011 to December 2014, in order to share the effectiveness of the two VR techniques.

\section{METHODS}

\section{Study design}

This study included 33 patients who underwent BCS in the Department of Breast Surgery, followed by TDAP flap $(n=14)$ or LICAP $(n=19)$ in the Department of Plastic and Reconstructive Surgery at our hospital from January 2011 to December 2014. To exclude any bias related to the surgical technique, lumpectomy and breast reconstruction were performed by the same surgeons, respectively, in each department. Details pertaining to underlying disease, age, body mass index, weight, surface area of skin excised, adjuvant treatment, and complications were collected from patients' medicalrecords for analysis and comparison. Patient satisfaction surveys were conducted 12 months following breast reconstruction and radiation treatment by using the Kyungpook National University Hospital modification of the Breast- $Q$ (Table 1). Cosmetic outcomes were measured by combining self-reported patient satisfaction with blinded evaluation performed by three plastic surgeons.

We conducted this study in compliance with the principles of the Declaration of Helsinki. Informed written consent was obtained from all patients.

\section{Surgical technique}

All surgical procedures were conducted under general anesthesia. BCS was followed by immediate breast reconstruction if the resected margins and frozen biopsy of sentinel lymph nodes were free of tumor, and if not, re-excision (approximately $2 \mathrm{~cm}$ ) was performed followed by total excision of axillary lymph

Table 1. The Kyungpook National University Hospital (KNUH) modification of the Breast-0.

\begin{tabular}{|c|c|c|c|c|c|}
\hline Items & $\begin{array}{c}\text { Very } \\
\text { satisfied }\end{array}$ & Satisfied & Neutral & Unsatisfied & $\begin{array}{c}\text { Very } \\
\text { unsatisfied }\end{array}$ \\
\hline Overall, are you satisfied with your breast reconstruction? & 5 & 4 & 3 & 2 & 1 \\
\hline Are you satisfied with breast symmetry achieved after reconstruction? & 5 & 4 & 3 & 2 & 1 \\
\hline Are you satisfied with the size of your breast after reconstruction? & 5 & 4 & 3 & 2 & 1 \\
\hline Are you satisfied with the shape of your breast after reconstruction? & 5 & 4 & 3 & 2 & 1 \\
\hline Are you satisfied with how your breasts feel after reconstruction? & 5 & 4 & 3 & 2 & 1 \\
\hline Are you satisfied with the level of pain you had to endure after reconstruction? & 5 & 4 & 3 & 2 & 1 \\
\hline Are you satisfied with the scar resulted after breast reconstruction? & 5 & 4 & 3 & 2 & 1 \\
\hline Are you satisfied with the donor site scar (back, flank or abdomen)? & 5 & 4 & 3 & 2 & 1 \\
\hline Are you satisfied with the donor site pain (back, flank or abdomen)? & 5 & 4 & 3 & 2 & 1 \\
\hline Have you experienced a loss of confidence or self-esteem after breast reconstruction? & 5 & 4 & 3 & 2 & 1 \\
\hline Are you satisfied with your sexual attractiveness after breast reconstruction? & 5 & 4 & 3 & 2 & 1 \\
\hline
\end{tabular}


nodes to ensure oncological safety.

\section{Preoperative assessment}

Preoperatively, 3-dimensional (3D) chest computed tomography (CT) angiography was performed to assist in the choice of the breast reconstruction technique and to ensure vessel conditions for TDAP and LICAP flaps. Subsequently, breast size, tumor size and the estimated location of the defect were measured to design the flap. The incision for BCS was designed to allow the best possible access and cosmetic results. A pinch test was performed to evaluate the amount of skin and fat to be harvested from the donor site, and the flap was designed based on the volume and mass of tissue available. The donor incision was made either horizontally, along the lines of skin tension, or vertically, in consultation with the patient to ensure that the scar was not visible. The flap was designed for different flap techniques rather than just one, as a way of preparing for possible variations in the excision volume.

\section{Thoracodorsal artery perforator flap}

The thoracodorsal artery arises from the subscapular artery and divides into a horizontal and a descending branch. The latter further divides into 2-3 cutaneous perforators that are located $8-10 \mathrm{~cm}$ inferior to the posterior axillary fold, and $2-3 \mathrm{~cm}$ posterior to the lateral border of the latissimus dorsi (LD) muscle (Fig. 1A). The perforators located at this site were marked on the skin by using a unidirectional Doppler probe. A pinch test was then performed to determine the feasibility of achieving primary closure. Finally, donor skin markings were made within the permissible skin paddle, based on the estimated flap volume. The donor incision was planned to ensure that the resultant scar

\section{Fig. 1. Thoracodorsal artery perforator (TDAP) flap}

(A) Path of the thoracodorsal artery and TDAP flap design; (B) Flap insetted to breast defect.
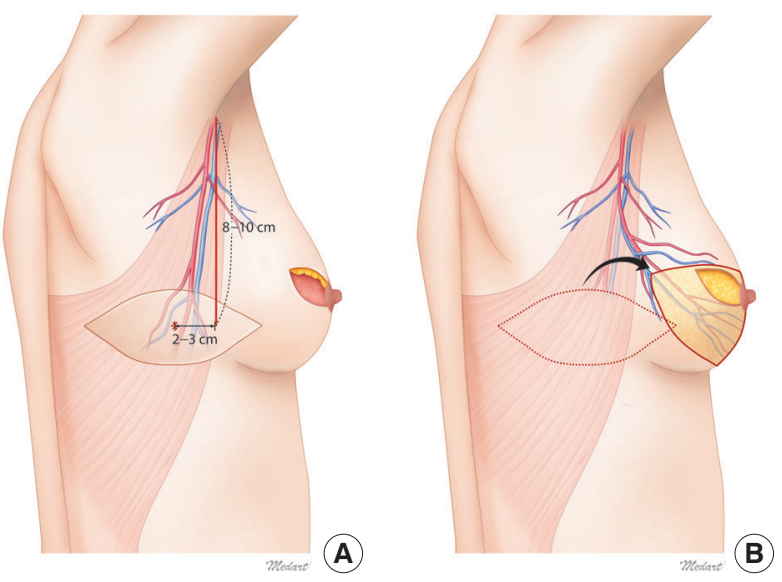

was hidden under the bra strap. Since these perforators tend to be relatively short, they were dissected from the muscle until the thoracodorsal artery and vein were met. In cases where a long pedicle and angle rotation was required, dissection was continued superiorly till the axillary area (Fig. 1B). A subcutaneous tunnel was created between the anterior border of the LD muscle and the defect, through which the flap was passed before being transposed into the breast defect. During each step, extreme care was taken to avoid damaging the perforators.

\section{Lateral Intercostal artery perforator flap}

The lateral intercostal artery perforators of the posterior intercostal artery are located $3 \mathrm{~cm}$ anterior to the anterior border of the LD muscle and pierce the serratus anterior muscle to supply the overlying skin and subcutaneous soft tissues (Fig. 2A). The perforators usually arise from the intercostal spaces numbered 5-8, with the most dominant perforator arising from the eighth intercostal space [15]. The incision was planned to position the resultant scar along the inframammary fold. While arranging the perforators to be titled anteriorly at the position similar to that of the TDAP flap design, the design including the respective LICAP perforator is performed (Fig. 2B). As the TDAP and LICAP flap designs were similar, they were interchangeably used if identification of the perforators for either of the flaps was difficult. The blood flow within the perforators is induced by the choke vessels between the segmental intercostal perforating branches that form subcutaneous arcades. Therefore, it is difficult to cover a distal area or a large defect when the perforator length is short and narrow. Since a reliable perforator may be easily missed, the LICAP flap was designed carefully to avoid complications such as fat necrosis. The elevated flap was tun-

\section{Fig. 2. Lateral Intercostal artery perforator (LICAP) flap}

(A) Path of the posterior intercostal artery and LICAP flap design; (B) Flap insetted to breast defect.
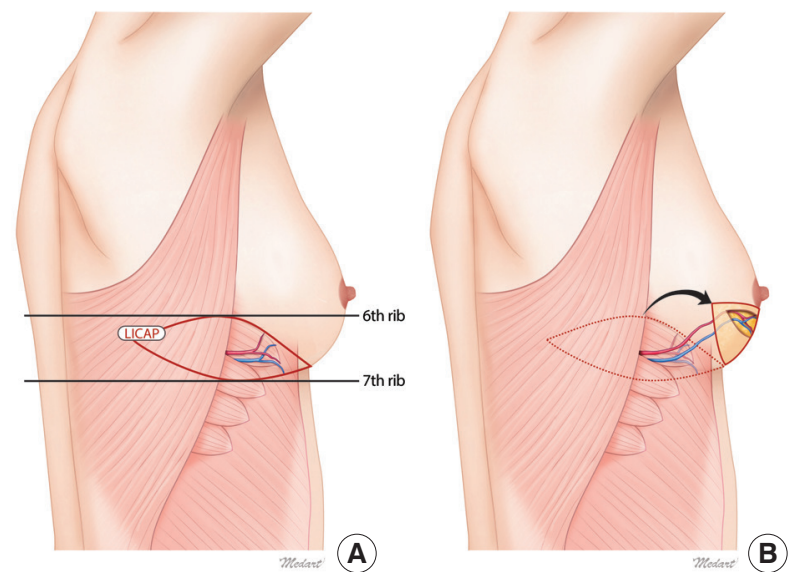
neled and transposed into the defect by using a propeller flap method. In case wherein a skin paddle was not required, the perforator was dissected with the fascial layer, and the flap was positioned by using a turnover method to achieve a stable circulation.

\section{RESULTS}

Thirty-three patients underwent BCS and partial breast reconstruction: 14 and 19 patients received the TDAP and LICAP flap, respectively.

The mean age of the subjects was $47.21 \pm 7.76$ years and the mean BMI was $23.38 \pm 3.70 \mathrm{~kg} / \mathrm{m}^{2}$. The underlying diseases identified were hypertension $(n=7)$ and diabetes $(n=4)$. The mean weight of excised tumors was $71.18 \pm 22.86 \mathrm{~g}$, and a positive margin on frozen biopsy was detected in three cases (9.1\%). The mean procedure time (breast surgery and plastic surgery) was $249.3 \pm 40.1$ minutes; the average length of hospital stay was $10.9 \pm 3.1$ days; and the average follow-up period per patient was $25.2 \pm 8.69$ months (Table 2).

The resected tumors were found to be invasive ductal carcino$\mathrm{ma}$ (IDC) $(\mathrm{n}=31)$ or ductal carcinoma in situ (DCIS) $(\mathrm{n}=2)$ on histopathology. Patients were categorized as one of four cancer-stages: Stage $0(\mathrm{n}=2)$, Stage IA $(\mathrm{n}=11)$, Stage IIA $(\mathrm{n}=$ $11)$, and Stage IIB $(n=9)$. Twenty-one subjects underwent adjuvant chemotherapy after surgery, and all the subjects received adjuvant radiotherapy (Table 3 ).

The choice of flaps in the different patients in presented in Table 4 .

Four patients had complications including wound disruption (TDAP flap, 2 cases; LICAP flap, 2 cases) that required major revision. The necrosis in these patients developed at the edge opposite to the location of the perforators. Linear necrosis developed in eight cases (TDAP flap, 4 cases; LICAP flap, 4 cases) and healed secondarily. Four cases (TDAP flap, 1 case; LICAP flap, 3 cases) required additional treatment for fat necrosis. There were no complications in 17 patients (TDAP flap, 7 cases; LICAP flap, 10 cases) (Table 5).

In the patient and physician satisfaction survey conducted 12 months postoperatively, 15 patients (46\%) (TDAP flap, 50\%; LICAP flap, 42\%) and 12 patients (36\%) (TDAP flap, 36\%; LICAP flap, 42\%) out of 33 subjects rated their satisfaction as excellent and good, respectively (Table 5). However, there was no

Table 3 . Tumor characteristics of 33 patients with breast cancer who underwent partial breast reconstruction using TDAP, LICAP flap

\begin{tabular}{|lccc|}
\hline Variables & TDAP & LICAP & Total \\
\hline Type of carcinoma & & & \\
Invasive ductal carcinoma & $14(100)$ & $17(89.5)$ & $31(93.9)$ \\
$\quad$ Ductal carcinoma in situ & 0 & $2(10.5)$ & $2(6.1)$ \\
T stage & & & \\
Tis & 0 & $2(10.5)$ & $2(6.1)$ \\
T1 & $8(57.1)$ & $11(57.9)$ & $19(57.6)$ \\
T2 & $6(42.9)$ & $6(31.6)$ & $12(36.3)$ \\
N stage & & & \\
N0 & $8(57.1)$ & $11(57.9)$ & $19(57.6)$ \\
N1 & $6(42.9)$ & $8(42.1)$ & $14(42.4)$ \\
Overall stage & & & \\
0 & 0 & $2(10.5)$ & $2(6.1)$ \\
IA & $4(28.6)$ & $7(36.9)$ & $11(33.3)$ \\
IIA & $6(42.8)$ & $5(26.3)$ & $11(33.3)$ \\
IIB & $4(28.6)$ & $5(26.3)$ & $9(27.3)$ \\
Adjuvant chemotherapy & & & \\
Positive & $8(57.1)$ & $13(68.4)$ & $21(63.6)$ \\
Negative & $6(42.9)$ & $6(31.6)$ & $12(36.4)$ \\
Adjuvant radiation treatment & & & \\
Positive & $14(100)$ & $19(100)$ & $33(100)$ \\
Negative & 0 & 0 & 0 \\
Recurrence & 0 & 0 & 0 \\
\hline Values are presented as mean \pm standard deviation or number (\%). \\
TDAP, thoracodorsal artery flap; LICAP, lateral intercostal artery flap. \\
\hline
\end{tabular}

Table 2. Patients characteristics of patients with breast cancer who underwent partial breast reconstruction using TDAP, LICAP flap

\begin{tabular}{|lccc|}
\hline Variables & TDAP $(\mathbf{n = 1 4 )}$ & LICAP $(\mathbf{n = 1 9 )}$ & Total $(\mathbf{n = 3 3 )}$ \\
\hline Mean age $(\mathrm{yr})$ & $49.28( \pm 7.94)$ & $45.68( \pm 7.26)$ & $47.21( \pm 7.76)$ \\
Mean body mass index $\left(\mathrm{kg} / \mathrm{m}^{2}\right)$ & $22.99( \pm 3.01)$ & $23.67( \pm 4.11)$ & $23.38( \pm 3.70)$ \\
Underlying disease & & & \\
Hypertension & $2(14.2)$ & $5(26.3)$ & $7(21.2)$ \\
Well-controlled diabetes mellitus & $2(14.2)$ & $2(10.5)$ & $4(12.1)$ \\
Mean volume of excised breast weight (g) & $81.42( \pm 24.73)$ & $63.63( \pm 17.96)$ & $71.18( \pm 22.86)$ \\
Initial margin positivity & $1(7.1)$ & $2(10.5)$ & $3(9.1)$ \\
Mean operation time (min) & $267.3( \pm 35.3)$ & $241.2( \pm 42.4)$ & $249.3( \pm 40.1)$ \\
Mean hospital stay (day) & $11.8( \pm 2.6)$ & $10.2( \pm 3.3)$ & $10.9( \pm 3.1)$ \\
Mean follow-up period (mo) & $26.3( \pm 8.5)$ & $24.4( \pm 8.75)$ & $25.2( \pm 8.69)$ \\
\hline Values are presented as mean \pm standard deviation or number (\%). & & \\
TDAP, thoracodorsal artery flap; LICAP, lateral intercostal artery flap. & & \\
\hline
\end{tabular}


Table 4. Reconstruction characteristics of partial breast reconstruction using TDAP and LICAP flaps

\begin{tabular}{|c|c|c|c|c|c|}
\hline Case & $\begin{array}{l}\text { Tumor } \\
\text { site }\end{array}$ & $\begin{array}{l}\text { Tumor } \\
\text { location }\end{array}$ & $\begin{array}{l}\text { Reconstruction } \\
\text { technique }\end{array}$ & $\begin{array}{l}\text { Flap skin } \\
\text { paddle } \\
\text { size }(\mathrm{cm})\end{array}$ & $\begin{array}{c}\text { Flap } \\
\text { weight (g) }\end{array}$ \\
\hline 1 & Left & UOQ, LOQ & TDAP & $6 \times 17$ & 120 \\
\hline 2 & Left & LOQ & TDAP & $8 \times 18$ & 90 \\
\hline 3 & Left & LOQ & LICAP & $4 \times 10$ & 90 \\
\hline 4 & Left & UOQ, LOQ & TDAP & $7 \times 20$ & 130 \\
\hline 5 & Left & LOQ & TDAP & $7 \times 20$ & 80 \\
\hline 6 & Left & LOQ & TDAP & $7 \times 15$ & 70 \\
\hline 7 & Right & LOQ & LICAP & $4 \times 10$ & 65 \\
\hline 8 & Right & LOQ & LICAP & $6 \times 16$ & 105 \\
\hline 9 & Left & UOQ, LOQ & TDAP & $5 \times 15$ & 110 \\
\hline 10 & Left & LOQ & LICAP & $4 \times 12$ & 90 \\
\hline 11 & Left & LOQ & LICAP & $6 \times 17$ & 80 \\
\hline 12 & Left & LOQ & LICAP & $4 \times 12$ & 80 \\
\hline 13 & Right & LOQ & LICAP & $6 \times 17$ & 120 \\
\hline 14 & Left & LOQ & LICAP & $4 \times 12$ & 70 \\
\hline 15 & Right & LOQ & LICAP & $4 \times 12$ & 70 \\
\hline 16 & Right & LOQ & TDAP & $4 \times 12$ & 70 \\
\hline 17 & Left & UOQ, LOQ & TDAP & $6 \times 18$ & 110 \\
\hline 18 & Left & LOQ & LICAP & $6 \times 15$ & 80 \\
\hline 19 & Right & LOQ & LICAP & $4 \times 12$ & 75 \\
\hline 20 & Right & LOQ & LICAP & $5 \times 15$ & 110 \\
\hline 21 & Left & UOQ, LOQ & TDAP & $6 \times 18$ & 150 \\
\hline 22 & Left & $\mathrm{LOQ}$ & TDAP & $5 \times 15$ & 90 \\
\hline 23 & Left & LOQ & TDAP & $8 \times 18$ & 110 \\
\hline 24 & Left & LOQ & TDAP & $5 \times 15$ & 75 \\
\hline 25 & Left & LOQ & LICAP & $5 \times 15$ & 110 \\
\hline 26 & Left & LOQ & LICAP & $5 \times 15$ & 80 \\
\hline 27 & Left & LOQ & LICAP & $5 \times 15$ & 60 \\
\hline 28 & Left & LOQ & TDAP & $5 \times 12$ & 120 \\
\hline 29 & Left & $\mathrm{LOQ}$ & LICAP & $5 \times 17$ & 60 \\
\hline 30 & Left & LOQ & LICAP & $5 \times 20$ & 100 \\
\hline 31 & Left & LOQ & LICAP & $5 \times 15$ & 80 \\
\hline 32 & Right & LOQ & LICAP & $4 \times 12$ & 60 \\
\hline 33 & Right & UOQ, LOQ & TDAP & $5 \times 15$ & 120 \\
\hline
\end{tabular}

TDAP, thoracodorsal artery flap; LICAP, lateral intercostal artery flap; U, upper; 0 , outer; $Q$, quadrant; L, lower.

significant difference in the patient satisfaction levels between the two flaps.

\section{DISCUSSION}

Breasts are considered as symbols of femininity, and play a crucial role in the mental, social, and sexual health of a woman. Surgical efforts to reconstruct breasts to achieve their near-normal shape by using autologous tissue or breast implants have contributed to the advances in mastectomy techniques. In 1995, Fisher et al. [16] reported similar local recurrence and survival rates in breast cancer patients, with tumors $4 \mathrm{~cm}$ or smaller and without axillary lymphatic invasion, who received either TM or BCS with RT. Since then, BCS with RT has emerged as the treat-

\section{Table 5. Cosmetic outcome and complications}

\begin{tabular}{|c|c|c|c|}
\hline $\begin{array}{l}\text { Combined assessment of } \\
\text { surgeons and patients }\end{array}$ & n (\%) & Complications & n (\%) \\
\hline $\operatorname{TDAP}(n=14)$ & & $\operatorname{TDAP}(n=14)$ & \\
\hline Excellent & $7(50)$ & Major revision & $2(14)$ \\
\hline Good & $5(36)$ & Linear necrosis & $4(29)$ \\
\hline Fair & $2(14)$ & Fat necrosis & $1(7)$ \\
\hline Poor & 0 & None & $7(50)$ \\
\hline $\operatorname{LICAP}(n=19)$ & & $\operatorname{LICAP}(n=19)$ & \\
\hline Excellent & $8(42)$ & Major revision & $2(11)$ \\
\hline Good & $8(42)$ & Linear necrosis & $4(21)$ \\
\hline Fair & $3(15)$ & Fat necrosis & $3(16)$ \\
\hline Poor & 0 & None & $10(52)$ \\
\hline
\end{tabular}

ment of first choice for patients with early stage breast cancer.

As an extended concept of BCS, oncoplastic breast surgery focuses on reconstructing the breast after lumpectomy by using residual breast tissue and, in many cases, adjacent tissue. It combines the advantages of adequate tumor margins and better cosmetic outcomes. The factors that determine the cosmetic outcomes following BCS include the volume of excised breast tissue, tumor location, breast size, radiotherapy, re-excision, and postoperative scar size. Among them, the volume of excised tissue is the most important. In the instance of residual breast tissue being inadequate for satisfactory breast reconstruction, an LD flap is a popular option owing to the coverage and tissue volume it provides. However, in addition to donor site morbidity, it may also be complicated by damage to the muscles important for shoulder movement. For Korean women with small- to moderate-sized breasts, partial breast reconstruction techniques based on perforator flaps have proven efficacy. These flaps were first described by Hamdi et al., and subsequently underwent several modifications $[7,8,10]$. Plastic surgeons in our hospital have devised algorithms to aid in the choice of breast reconstruction techniques for patients with small- to moderate-sized breasts $[7,8]$. Volume displacement (VD) techniques are not feasible in these patients owing to a lack of adequate residual breast tissue. In the presence of large defect-to-breast ratios, VR techniques are required. Experience has taught us that the defect-to-breast volume ratio is more effective than a simple definition of moderate-sized defect based on weight or size, in assessing these patients. Even if patients with small- to moderatesized breasts are classified as having a moderate-sized defect, their excised volumes are not significant. This implies that the use of a technique such as LD flap in these patients may result in significant donor morbidity. In such cases, a pedicled perforatorbased TDAP or LICAP flap may provide better clinical out- 
comes.

A TDAP flap can provide coverage of large defects over the upper arm and axillary areas. It is a fasciocutaneous flap that uses the perforators originating from the descending branch of the thoracodorsal artery. Its advantages include a preserved LD muscle, reduced donor site complications, shortened recovery period, and adequate coverage for wide defects. However, this flap is limited by the difficulty in locating the perforator vessels with Doppler studies since the perforators are very small. The dissection of the perforators, and therefore the use of this flap, requires greater surgical proficiency (Fig. 3) [12,17].

The LICAP flap has gained popularity as a perforator flap technique with similar advantages and disadvantages as the TDAP flap. Both flaps require a change in position during surgery, despite the location of perforators being further anterior for the LICAP flap than the TDAP flap. In addition, the skin markings are similar for both flaps. On the positive side, the scar following LICAP flap reconstruction is less visible as it is hidden under the arm, and the reconstructed breast is more natural in appearance owing to the similarity in skin texture between the flap and the breast. Also, the LICAP flap allows for easier identification of perforators through anterior dissection, and therefore may be used as an alternative to the TDAP flap when its perforators cannot be easily identified. However, LICAP flap perfora-

\section{Fig. 3. Case 1: right-sided breast cancer}

Breast-conserving surgery in a 47-year-old woman involving removal of the tumor (98 g), followed by TDAP flap-based breast reconstruction. (A) Preoperative photograph. (B) Dissected TDAP flap. (C) Follow-up photograph 12 months after surgery. TDAP, thoracodorsal artery perforator.
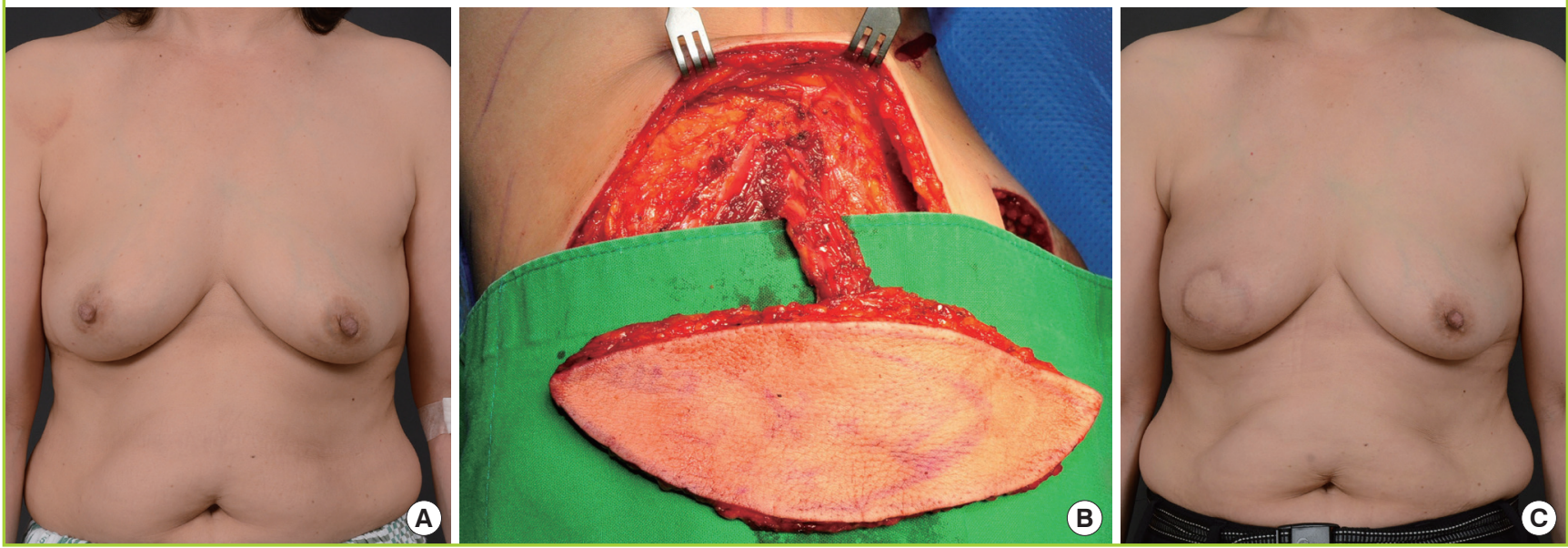

\section{Fig. 4. Case 2: right-sided breast cancer}

Breast-conserving surgery in a 43-year-old woman involving removal of the tumor $(75 \mathrm{~g})$, followed by LICAP flap-based (propeller and tunneling method) breast reconstruction. (A) Preoperative photograph. (B) Dissected LICAP flap. (C) Follow-up photograph 33 months after surgery. LICAP, lateral intercostal artery perforator.
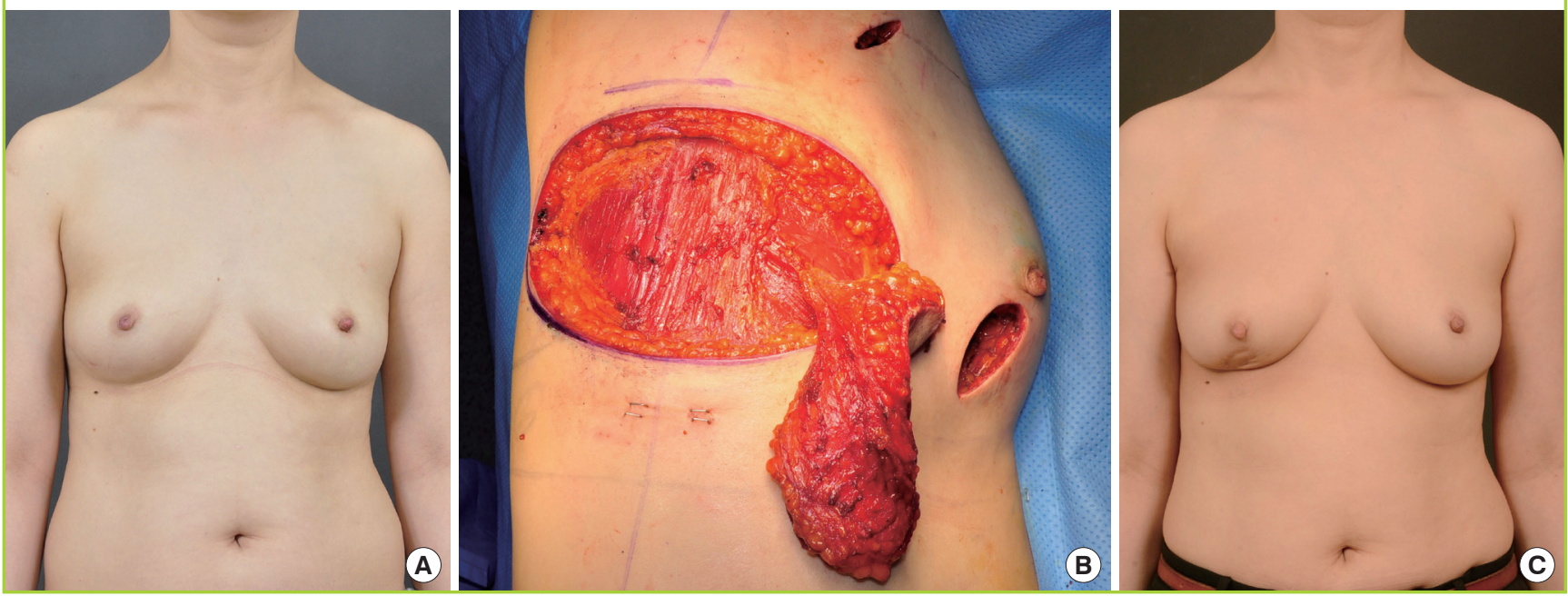
tors are short in length, making it useful for the coverage of defects in the lower outer quadrant of the breast alone. The flap is also at a higher risk of developing fat necrosis and flap necrosis in the areas farthest to the perforators $[11,15]$.

There are two methods of transposing an elevated LICAP flap into a defect. In cases where a skin paddle is required because of excision of excess breast skin, after securing the maximum possible length of the pedicle, the perforator is skeletonized and the LICAP flap is rotated to the desired direction by using the propeller flap method (Fig. 4). However, this technique is associated with a risk of fat necrosis owing to venous congestion, and major revisions may be required. In our hospital, fat necrosis developed in three cases when the LICAP flap was positioned in the method described above. To overcome this complication, we now avoid excessive skeletonization in cases wherein a skin paddle is not required, dissect the perforator along with the adjacent fascia, and transpose the flap into the defect by using a turnover method. This approach prevents excessive tension on the perforator and reduces the incidence of venous congestion, and therefore, the risk of complications such as fat necrosis [18].

Revision operation was required in two cases for each flap technique wherein complications occurred in the area farthest from the perforators. In addition, the incidence of fat necrosis was higher with the use of LICAP flap ( 3 cases) than the TDAP flap ( 1 case). These complications may be associated with the differences in blood flow and the degree of flap tension based on flap thickness and their laterality.

Thirty-three patients with breast cancer underwent partial mastectomy and immediate oncoplastic breast surgery with TDAP or LICAP flaps. All subjects had small- to moderatesized breasts and moderate-sized defects. In the patient satisfaction survey conducted 12 months following breast reconstruction and radiotherapy, more than $80 \%$ of subjects, 12 (86\%) with TDAP flap and 16 (84\%) with LICAP flap, were satisfied with the outcome (Table 5).

These results showing more than $80 \%$ cosmetic satisfaction were similar to the results reported in a previous article by our institution on breast reconstruction using an LD flap [7]. Therefore, when possible, breast reconstruction using a perforator flap appears to be more useful. The overall oncological outcomes were also satisfactory (Table 3 ).

TDAP and LICAP flaps showed good outcomes in terms of cosmetic and oncological aspects. In choosing between these two flaps, the decision is influenced by the location and size of the defect but may also depend on the experience of the surgeon. Therefore, if the plan involves the use of a perforator flap, the preparations and design of both methods should be implemented preoperatively.
When using a perforator flap, if careful attention is paid during the harvesting process and the procedure is supported by the proficiency of the surgeon, and the experience of the surgeon in choosing the reconstruction technique, then it can be a useful technique that lowers donor morbidity, as compared with a musculocutaneous flap such as a LD flap, which uses the main branch as the pedicle, while producing good cosmetic outcomes with similar complication rate. Therefore, on the basis of the 5-year experience of our institution, we believe that for patients with relatively small breasts and small-to-medium breast defects, oncoplastic breast surgery using a TDAP or LICAP flap may be a useful technique.

\section{CONFLICT OF INTEREST}

No potential conflict of interest relevant to this article was reported.

\section{PATIENT CONSENT}

The patient provided written informed consent for the publication and the use of their images.

\section{REFERENCES}

1. Zumsteg ZS, Morrow M, Arnold B, et al. Breast-conserving therapy achieves locoregional outcomes comparable to mastectomy in women with T1-2N0 triple-negative breast cancer. Ann Surg Oncol 2013;20:3469-76.

2. Veronesi U, Cascinelli N, Mariani L, et al. Twenty-year follow-up of a randomized study comparing breast-conserving surgery with radical mastectomy for early breast cancer. $\mathrm{N}$ Engl J Med 2002;347:1227-32.

3. Arndt V, Stegmaier C, Ziegler H, et al. Quality of life over 5 years in women with breast cancer after breast-conserving therapy versus mastectomy: a population-based study. J Cancer Res Clin Oncol 2008;134:1311-8.

4. Munhoz AM, Montag E, Arruda E, et al. Immediate conservative breast surgery reconstruction with perforator flaps: new challenges in the era of partial mastectomy reconstruction? Breast 2011;20:233-40.

5. Hamdi M, Van Landuyt K, Monstrey S, et al. Pedicled perforator flaps in breast reconstruction: a new concept. Br J Plast Surg 2004;57:531-9.

6. Yang JD, Lee JW, Kim WW, et al. Oncoplastic surgical techniques for personalized breast conserving surgery in breast cancer patient with small to moderate sized breast. J Breast Cancer 2011;14:253-61. 
7. Yang JD, Kim MC, Lee JW, et al. Usefulness of oncoplastic volume replacement techniques after breast conserving surgery in small to moderate-sized breasts. Arch Plast Surg 2012;39:489-96.

8. Yang JD, Lee JW, Cho YK, et al. Surgical techniques for personalized oncoplastic surgery in breast cancer patients with small- to moderate-sized breasts (part 2): volume replacement. J Breast Cancer 2012;15:7-14.

9. Hamdi M, De Frene B. Pedicled perforator flaps in breast reconstruction. Semin Plast Surg 2006;20:73-8.

10. Hamdi M, Rasheed MZ. Advances in autologous breast reconstruction with pedicled perforator flaps. Clin Plast Surg 2012;39:477-90.

11. Hamdi M, Van Landuyt K, de Frene B, et al. The versatility of the inter-costal artery perforator (ICAP) flaps. J Plast Reconstr Aesthet Surg 2006;59:644-52.

12. Santanelli F, Longo B, Germano S, et al. Total breast reconstruction using the thoracodorsal artery perforator flap without implant. Plast Reconstr Surg 2014;133:251-4.

13. D’Aniello C, Grimaldi L, Barbato A, et al. Cosmetic results in
242 patients treated by conservative surgery for breast cancer. Scand J Plast Reconstr Surg Hand Surg 1999;33:419-22.

14. Clough KB, Cuminet J, Fitoussi A, et al. Cosmetic sequelae after conservative treatment for breast cancer: classification and results of surgical correction. Ann Plast Surg 1998;41:471-81.

15. Hamdi M, Spano A, Van Landuyt K, et al. The lateral intercostal artery perforators: anatomical study and clinical application in breast surgery. Plast Reconstr Surg 2008;121:38996.

16. Fisher B, Anderson S, Redmond CK, et al. Reanalysis and results after 12 years of follow-up in a randomized clinical trial comparing total mastectomy with lumpectomy with or without irradiation in the treatment of breast cancer. N Engl J Med 1995;333:1456-61.

17. Hamdi M, Salgarello M, Barone-Adesi L, et al. Use of the thoracodorsal artery perforator (TDAP) flap with implant in breast reconstruction. Ann Plast Surg 2008;61:143-6.

18. McCulley SJ, Schaverien MV, Tan VK, et al. Lateral thoracic artery perforator (LTAP) flap in partial breast reconstruction. J Plast Reconstr Aesthet Surg 2015;68:686-91. 